intestinal cancer development. Nature 2005:437:281-5.

30 Prokhortchouk A, Sansom O, Selfridge J, et al. Kaiso-deficient mice show resistance to intestinal cancer. Mol Cell Biol 2006;26:199-208.

31 Perreault N, Sackett SD, Katz JP, et al. Foxl1 is a mesenchymal modifier of Min in carcinogenesis of stomach and colon. Genes Dev 2005;19:311-5.

32 Oster H, Leitges M. Protein kinase $\mathrm{C}$ alpha but not PKCzeta suppresses intestinal tumor formation in ApcMin/+ mice. Cancer Res 2006;66:6955-63.

33 Wilson CL, Heppner KJ, Labosky PA, et al. Intestina tumorigenesis is suppressed in mice lacking the metalloproteinase matrilysin. Proc Natl Acad Sci U S A 1997:94:1402-7.

34 Sansom OJ, Mansergh FC, Evans MJ, et al. Deficiency of SPARC suppresses intestinal tumourigenesis in APC ${ }^{\mathrm{Min} /+}$ mice. Gut 2007:56:1410-14

35 Hanahan D, Weinberg RA. The hallmarks of cancer. Cell 2000; 100:57-70.

36 Framson PE, Sage EH. SPARC and tumor growth: where the seed meets the soil? I Cell Biochem 2004;92:679-90.

37 Murphy-Ullrich JE. The de-adhesive activity of matricellular proteins: is intermediate cell adhesion
38 Bradshaw AD, Sage EH. SPARC, a matricellular protein that functions in cellular differentiation and tissue response to injury. J Clin Invest 2001 ; 107:1049-54

39 Brekken RA, Puolakkainen P, Graves DC et al. Enhanced growth of tumors in SPARC null mice is associated with changes in the ECM. J Clin Invest 2003;111:487-95.

40 Sangaletti S, Stoppacciaro A, Guiducci C, et al. Leukocyte, rather than tumor-produced SPARC, determines stroma and collagen type IV deposition in mammary carcinoma. J Exp Med 2003; 198: 1475-85.

\title{
What is the role of $\mathrm{iFOBT}$ in screening for colorectal cancer?
}

\section{David F Ransohoff}

\section{Policy makers will need to consider if it has one, not only as an adjunct to gFOBT screening, but also as a primary screening test}

S creening for colorectal cancer (CRC) using gFOBT (guaiac based faecal occult blood test) has been shown in randomised controlled trials (RCTs) to reduce CRC mortality. ${ }^{1-3}$ gFOBT testing is endorsed as an option for CRC screening in the United States ${ }^{4-7}$ and is being implemented in the United Kingdom. People with a positive gFOBT receive colonoscopy to detect early cancers and advanced adenomas that, if untreated, might cause CRC mortality. Because gFOBT has a high rate of false positive results, however, gFOBT screening can incur substantial cost and use of colonoscopy resources. A method that could determine which people with a positive gFOBT have false positive results-and do not need colonoscopy-would make gFOBT screening more practical.

A study in this issue of $G u t^{8}$ Fraser et al (see $p$ 1415) shows that doing $\mathrm{iFOBT}$ (human haemoglobin immunochemical based FOBT) in people with a positive gFOBT will detect almost all clinically important lesions in gFOBT positive individuals, while reducing false positive results and the need for colonoscopy. In this study, both iFOBT and colonoscopy were done in those with a positive gFOBT, defined as one to four positive gFOBT ovals (people with five or six positive ovals were automatically referred for colonoscopy). The sensitivity of iFOBT among people with positive gFOBT was $95.9 \%$, while specificity was $59.2 \%$, resulting in a $30 \%$ reduction of colonoscopy use. The mechanism by which iFOBT achieves higher specificity than gFOBT presumably is elimination of false positives that occur in guaiac based testing from sources other than human haemoglobin, like diet.

Learning that iFOBT may have a role in a programme of gFOBT screening is practical and important, but it raises a larger question that will need to be sorted out over time. Should iFOBT replace gFOBT as the primary test in a programme of FOBT screening? The authors note that the unit cost for iFOBT is higher than for gFOBT, but that is only one consideration. ${ }^{8}$ Others include the increased benefit that might be achieved, additional costs (including false positives), and the public's willingness to pay for greater benefit.

The potential usefulness of iFOBT vs gFOBT screening depends largely on the "absolute sensitivity" of each FOBT, a question not addressed in this study that assessed iFOBT sensitivity only among those who already had a positive gFOBT. Used in this way, iFOBT can never do better than gFOBT.

What is the absolute sensitivity for each FOBT? Obtaining absolute sensitivity for any FOBT is logistically difficult, requiring administration of both the FOBT and a "gold standard" exam (like colonoscopy). In asymptomatic individuals the prevalence of CRC is so low, roughly 1-3 per 1000, that thousands of people need to be studied. In one report, gFOBT sensitivity for CRC was about $30 \%{ }^{9}$; in another it was $13 \% .^{10}$ The different results might be explained by use of centralised FOBT processing in the former study (as is also done in the current study, in RCTs, and in the UK screening programme being implemented), while in the latter study processing was done in each physician's office.

While these are low values for absolute sensitivity of gFOBT, they are high enough, as shown in RCTs, to reduce CRC mortality by $33 \%$ in a US $^{1}$ trial and by about $16 \%$ in two European trials. ${ }^{2}$ Some mortality reduction may be achieved by repeated application of FOBT in a programme of screening. gFOBT screening is considered not only an "option" for CRC screening, but when combined with sigmoidoscopy, it may be competitive, in terms of effectiveness, with a programme of colonoscopy screening. ${ }^{11}{ }^{12}$ This seeming paradox-that a less sensitive test like FOBT might be as effective or more effective than a "gold standard" test like colonoscopy—can be explained by considering how a "programme" of screening works. If a test with lower sensitivity at any one application is applied repeatedly over time in a programme of screening, it may result in a higher "programme" sensitivity than when a more sensitive test (like colonoscopy) is applied less frequently, because a more frequently applied test can detect fast growing CRC that would be missed by a less frequently applied test. ${ }^{11-13}$

If gFOBT is not simply "acceptable" but perhaps is even competitive with colonoscopy in some settings, then what would be the impact of a better FOBT? The absolute sensitivity of iFOBT in recent studies has been shown to be roughly $60 \%$ for $\mathrm{CRC}^{14-17}$ much higher than for gFOBT. Even without new RCT data to assess CRC mortality reduction, policy decisions may be based on data about the sensitivity and specificity of newer tests, existing RCT results, and modelling. ${ }^{7}$ Policy makers will need to consider whether iFOBT has a role not only as an adjunct to gFOBT screening, but also as a primary screening test.

Gut 2007;56:1343-1344.

doi: $10.1136 /$ gut.2007.124107

Correspondence to: David F Ransohoff, CB7080, University of North Carolina at Chapel Hill, 
Chapel Hill, NC 27599-7080, USA; ransohof@ med.unc.edu

Competing interest: None declared.

\section{REFERENCES}

1 Mandel JS, Bond JH, Church TR, et al. Reducing mortality from colorectal cancer by screening for fecal occult blood. N Engl J Med 1993;328:1365-71.

2 Hardcastle JD, Chamberlain JO, Robinson MHE, et al. Randomised controlled trial of faecal-occultblood screening for colorectal cancer. Lancet 1996;348: 1472-7.

3 Kronborg O, Fenger C, Olsen J, et al. Randomised study of screening for colorectal cancer with faecaloccult-blood test. Lancet 1996;348:1467-71.

4 Winawer S, Fletcher R, Rex D, et al. Colorectal cancer screening and surveillance: clinical guidelines and rationale-update based on new evidence. Gastroenterology 2003;124:544-60.

5 Screening for colorectal cancer: recommendation and rationale. Ann Intern Med 2002;137:129-31.
6 Smith RA, von Eschenbach AC, Wender R, et al. American Cancer Society Guidelines for the early detection of cancer: update of early detection guidelines for prostate, colorectal, and endometrial cancers. CA Cancer J Clin 2001;51:38-75.

7 Winawer SJ, Fletcher RH, Miller L, et al. Colorectal cancer screening: clinical guidelines and rationale. Gastroenterology 1997; 1 12:594-642.

8 Fraser CG, Mathew CM, Mowat NAG, et al. Evaluation of a card collection-based faecal immunochemical test in screening for colorectal cancer using a two-tier reflex approach. Gut 2007:56:1415-8.

9 Ahlquist DA, Wieand HS, Moertel CG, et al. Accuracy of fecal occult blood screening for colorectal neoplasia. A prospective study using Hemoccult and HemoQuant tests. JAMA 1993;269:1262-7.

10 Imperiale TF, Ransohoff DF, Itzkowitz SH, et al. Fecal DNA versus fecal occult blood for colorectalcancer screening in an average-risk population. N Engl J Med 2004;351:2704-14.

\section{Antioxidants in acute pancreatitis}

\section{D Johnson}

\section{Antioxidant supplements may be ineffective for the treatment or prevention of organ failure in predicted severe acute pancreatitis}

O xygen-derived free radicals are produced when a period of intracellular anaerobic respiration is followed by re-oxygenation. These extremely reactive radicals combine with a large number of different protein and lipid molecules causing tissue damage and cell injury. The normal defences against such free radical attack include the presence in the tissues of antioxidant compounds and pathways of metabolism. A lack of sufficient antioxidant reserve during times of increased production of free radicals leads to the state of oxidative stress.

There has been increasing awareness over the last 20 years of the role played by oxidative stress in many inflammatory illnesses. Acute pancreatitis is no exception and in several models it has been demonstrated that oxygen-derived free radicals are generated during acute pancreatitis. It has been suggested that free radical generation, or the inability to quench free radicals, is an important factor in the pathogenesis of acute pancreatitis. ${ }^{1}$ However, a careful experimental study suggested that oxidative stress alone cannot cause pancreatitis. ${ }^{2}$ It is much more plausible that oxidative stress may contribute to worsening of the local inflammatory changes after onset of pancreatitis. ${ }^{3-6}$ Some experimental studies suggest that antioxidant therapy can diminish tissue injury in acute pancreatitis. $^{37}$ It is also clear that oxidative mechanisms are an integral part of the inflammatory response and oxidative stress may contribute to pulmonary injury in severe acute pancreatitis. ${ }^{89}$ Oxidative stress is recognised as part of the pathophysiology of adult respiratory distress syndrome, $^{10}$ and there is experimental evidence that antioxidants can protect against lung injury in acute pancreatitis.

There is a convincing body of evidence that antioxidant blood levels diminish during severe acute pancreatitis, ${ }^{11}{ }^{12}$ and that supplements of antioxidants can prevent these falls in experimental ${ }^{3}$ and clinical $^{13}$ pancreatitis. However, the evidence of clinical benefit to support the therapeutic use of antioxidants is sparse and uncontrolled.

Given the supposed harmful nature of oxidative stress, and observation of antioxidant depletion in human pancreatitis, ${ }^{11-13}$, it has been postulated that the harmful effects of oxidative stress in this condition could be ameliorated by supplementation with naturally occurring antioxidants. Unfortunately there is little published evidence to support this theory. One randomised trial ${ }^{14}$ reported reduced frequency of attacks of recurrent acute pancreatitis, in a small study population
11 Pignone M, Saha S, Hoerger T, et al. Costeffectiveness analyses of colorectal cancer screening: a systematic review for the US Preventive Services Task Force. Ann Intern Med 2002;137:96-104

12 Pignone M, Russell L, Wagner J. Economic models of colorectal cancer screening in average-risk adults: workshop summary http://www.nap.edu/ catalog/1 1228.html, Washington, DC: National Academies Press 2005.

13 Ransohoff DF. Colon cancer screening in 2005: status and challenges. Gastroenterology 2005; 128:1685-95.

14 Allison JE. Colon cancer screening guidelines 2005: the fecal occult blood test option has become a better FIT. Gastroenterology 2005;129:745-8.

15 Morikawa T, Kato J, Yamaji Y, et al. A comparison of the immunochemical fecal occult blood test and total colonoscopy in the asymptomatic population. Gastroenterology 2005;129:422-8.

16 Levi Z, Rozen P, Hazazi R, et al. A quantitative immunochemical fecal occult blood test for colorectal neoplasia. Ann Intern Med 2007; 146:244-55.
(20 patients). This study made no observations relevant to the treatment of patients with severe acute pancreatitis. A case controlled series from Manchester (where the treatment rationale was developed) demonstrated that although antioxidant supplements could indeed prevent the fall in blood levels seen in severe acute pancreatitis, there was no observed effect on clinical outcome. ${ }^{13}$

Until now, there has been no reliable randomised trial that investigates the use of antioxidants to reduce the severity of complications in acute pancreatitis. In this issue, Siriwardena and colleagues ${ }^{15}$ report just such a randomised controlled trial (see page 1439).

Siriwardena et al. ${ }^{15}$ report a trial that carefully selected patients who might be expected to benefit from antioxidant treatment. They were recruited to the study within 72 hours of onset of pancreatitis and they had an APACHE-II score $>8$. That is, only patients with predicted severe acute pancreatitis were included, and treatment was started as early as possible. These patients are relatively few, and recruitment in three hospitals extended over 41 months. Interim analyses were conducted by a trial statistician at planned annual intervals and patients were treated according to the UK National Guidelines for the Management of Acute Pancreatitis. This trial therefore focuses on the patients most at risk, who were managed to a high standard of care. The authors are to be congratulated on achieving a relatively low mortality rate, below $10 \%$, for these potentially seriously ill patients.

Patients were randomised to receive a placebo injection or a mixture of antioxidants (n-acetylcysteine, selenium, vitamin C), by intravenous injection for 1 week. The two groups were well matched except that the active treatment group were older. 